\title{
Câncer na adolescência: as dificuldades enfrentadas para o desenvolvimento social e pessoal
}

\author{
Cancer in adolescence: the difficulties faced for social and personal development
}

Cáncer en la adolescencia: las dificultades del desarrollo social y personal

Beatriz Okuma da Costa ${ }^{1 *}$, Caroline Giusti Picca Sartori ${ }^{1}$, Gabriela Silva Kassawara ${ }^{1}$, Marília Narciso Soares ${ }^{1}$, Nayara dos Reis Valões ${ }^{1}$, Fábio Scaramboni Cantinelli².

\section{RESUMO}

Objetivo: Descrever as principais dificuldades enfrentadas e a influência destas na qualidade de vida do adolescente com câncer. Métodos: Trata-se de um estudo de natureza exploratório-descritivo e de corte transversal, conduzido com 21 pacientes, de ambos os sexos, com idade entre 12 e 17 anos, em tratamento oncológico no Instituto de Tratamento do Câncer Infantil. Os pacientes foram avaliados quanto à qualidade de vida durante o tratamento do câncer através da aplicação de questionário WHOQOL-bref. Os dados obtidos foram analisados através de ferramentas desenvolvidas e disponibilizadas pelo próprio grupo WHOQOL. O estudo foi aprovado por Comitê de Ética em Pesquisa. Resultados: A amostra, com média de idade de 14,6 anos, apresentou maioria feminina (52,4\%). Desta, $69 \%$ se enquadram em uma qualidade de vida boa a regular, enquanto apenas $31 \%$ necessitam de melhora. Dos domínios analisados, o domínio Meio Ambiente é o que possui maior média, enquanto o de Relações Sociais é o de menor média e o único classificado como "precisa melhorar". Conclusão: A maioria dos participantes deste estudo se enquadram em uma avaliação regular, mostrando a necessidade de mudanças para promover uma atenção à saúde além da habilidade científica e técnica.

Palavras-chave: Desenvolvimento do adolescente, Psico-oncologia, Qualidade de vida.

\begin{abstract}
Objective: To describe the main difficulties faced and their influence on the quality of life of adolescents with cancer. Methods: This was a cross-sectional exploratory-descriptive study conducted with 21 patients of both genders, aged between 12 and 17 years, undergoing oncological treatment at the Institute for Childhood Cancer Treatment. The patients were evaluated regarding their quality of life during cancer treatment by applying the WHOQOL-bref questionnaire. The data obtained were analyzed using tools developed and made available by the WHOQOL group itself. The study was approved by the Research Ethics Committee. Results: The sample, with an average age of 14.6 years, presented a female majority (52.4\%). Of these, $69 \%$ fit into a good to fair quality of life, while only $31 \%$ need improvement. Of the domains analyzed, the Environment domain has the highest average, while Social Relations is the one with the lowest average and the only one classified as "needs improvement". Conclusion: Most of participants in this study fall into a regular assessment, showing the need for changes to promote health care beyond scientific and technical ability.
\end{abstract}

Keywords: Adolescent development, Psycho-oncology, Quality of life.

1 Universidade de Mogi das Cruzes (UMC), São Paulo - SP. *E-mail: beatrizokuma@gmail.com

2 Instituto do Câncer do Estado de São Paulo (ICESP), São Paulo - SP.

SUBMETIDO EM: 1/2022

ACEITO EM: 1/2022

PUBLICADO EM: 2/2022 


\section{RESUMEN}

Objetivo: Describir las principales dificultades a las que se enfrentan y la influencia de éstas en la calidad de vida del adolescente con cáncer. Métodos: Se trata de un estudio de naturaleza exploratoria-descriptiva y de corte transversal, realizado con 21 pacientes de ambos sexos, con edad entre los 12 y los 17 años, en tratamiento oncológico en el Instituto para el Tratamiento del Cáncer Infantil. Los pacientes fueron evaluados en cuanto a la calidad de vida durante el tratamiento del cáncer mediante la aplicación del cuestionario WHOQOL-bref. Los datos obtenidos fueron analizados mediante herramientas desarrolladas y puestas a disposición por el propio grupo WHOQOL. El estudio fue aprobado por el Comité de Ética de la Investigación. Resultados: La muestra, con una edad media de 14,6 años, presentaba una mayoría femenina $(52,4 \%)$. De estos, el $69 \%$ se ajusta a una buena calidad de vida normal, mientras que solo el $31 \%$ necesita mejorar. De los dominios analizados, el dominio Entorno es el que tiene la media más alta, mientras que Relaciones Sociales es el que tiene la media más baja y el único clasificado como "necesita mejorar". Conclusión: La mayoría de los participantes en este estudio entran en una evaluación regular, lo que demuestra la necesidad de cambios para promover la atención sanitaria más allá de la capacidad científica y técnica.

Palabras clave: Desarrollo del adolescente, Psicooncología, Calidad de vida.

\section{INTRODUÇÃO}

Segundo Instituto Nacional do Câncer (INCA) (2015), o câncer consiste em um conjunto de doenças que tem em comum a proliferação de células anormais que ocorre de forma descontrolada e invadem os tecidos e órgãos podendo se espalhar para diversas partes do corpo, o que determinam as metástases.

Diferente do que ocorre nos adultos, o câncer infantojuvenil, que compreende a doença que acomete indivíduos entre 0 e 19 anos, afeta principalmente células de natureza embrionária, geralmente, células do sistema sanguíneo e os tecidos de sustentação, o que proporciona melhor resposta aos tratamentos atuais, na maioria dos casos (INCA, 2021).

Alguns aspectos relacionados ao estilo de vida, como alcoolismo, sedentarismo, obesidade e alimentação não balanceada exercem papel importante em muitos tipos de câncer em adultos. Porém, o câncer na criança e no adolescente parece não ter muito influência uma vez que esses fatores de risco geralmente precisam de anos para influenciar no risco aumentado de câncer. Em contrapartida, fatores ambientais, como exposição à radiação, e ainda exposição dos pais, como o fumo, foram associados a cânceres infantis, mas ainda é necessário mais estudo para determinar com certeza essa associação (AMERICAN CANCER SOCIETY (ACS), 2019a).

Segundo a última estimativa publicada pelo Instituto Nacional de Câncer o número de casos novos de câncer esperados para cada ano do triênio 2020-2022 será de 625 mil casos novos de câncer, porém desses, apenas 8.460 casos estão dentro da faixa etária de $0-19$ anos, o que representa 1,35\% de todos os cânceres registrados no Brasil e um risco estimado de 138, 44 casos novos por milhão. Dos casos de câncer infantojuvenil 4.310 são do sexo masculino e de 4.150 no feminino (INCA, 2019a).

O câncer na adolescência é considerado raro se comparado à incidência em adultos com uma pequena proporção da carga global do câncer (FELICIANO SVM, et al., 2018). Porém, por outro lado, demanda um maior cuidado por parte dos profissionais da saúde, uma vez que, a adolescência é uma fase de transformação e formação, ou seja, é a etapa de transição entre o ser infantil e o ser adulto, onde ocorre a construção do indivíduo psicologicamente e socialmente, sendo essa influenciada pela cultura, familiares, vínculos afetivos e meio social (DUARTE VI e GALVÃO Al, 2014).

Os primeiros desafios da trajetória têm início já na busca pelo diagnóstico e a confirmação do mesmo, uma vez que essa patologia está totalmente ligada com dor, rompimento do desenvolvimento, alterações corporais advindas da doença, incerteza de cura e até a morte. Dessa forma, a primeira reação é a de negação, onde se associa a recusa ao diagnóstico e raiva caracterizada por inconformismo pela doença (FERENSOVICZ JMR, 2020). 
Mesmo após o diagnóstico e a discussão do tratamento com a família, devido ao longo período de incertezas e de procedimentos dolorosos que a criança passou para confirmação do diagnóstico, o câncer é visto como algo assustador, que é capaz de realizar mudanças, desordens e manifestações nunca antes vivenciadas tanto pela criança ou adolescente quanto pela família, assim, suscita uma gama de sentimentos com forte impacto emocional como desesperança, incerteza, espanto e temor (ANJOS C, et al., 2015).

O câncer não é uma doença que atinge apenas o indivíduo, mas é uma doença social, ou seja, interfere a vida de todos em sua volta, desde as dificuldades econômicas que todo o tratamento pode causar, o medo de contar para as pessoas a fim de evitar comentários inadequados, surgimento de conflitos dentro dos membros da família e mudanças dos hábitos e costumes (FARINHAS VG, et al., 2013).

Para o doente, esse processo de adoecimento traduz em um período de rápidas e acentuadas transformações, pois, além das mudanças no ambiente familiar e na rotina, com hospitalizações que o distanciam do seu meio social, tem-se ainda a dor causada pelos tratamentos, algumas vezes invasivos, e ainda as mudanças corporais que prejudicam sua autoestima, como a alopecia, que é um dos efeitos colaterais mais devastadores do tratamento (VILLA D, 2019).

Visando a diminuição do impacto dessas possíveis alterações físicas, emocionais e sociais, e no intuito de proporcionar uma maior qualidade de vida, a oncologia surge como especialidade. Nesta perspectiva, a mensuração da qualidade de vida torna-se importante para avaliar os resultados do tratamento. Dessa forma, com tantas mudanças e influências que o diagnóstico do câncer pode ter na vida de um adolescente, fase de tantas mudanças biológicas, cognitivas, emocionais e sociais, o estudo objetivou definir as dificuldades enfrentadas e avaliar a qualidade de vida além dos aspectos sociodemográficos dos pacientes oncológicos em tratamento em um Instituto de tratamento no estado de São Paulo.

\section{MÉTODOS}

Trata-se de um estudo de natureza exploratório-descritivo, de abordagem quali-quantitativa de corte transversal que buscou informações sobre a percepção do tratamento pelo adolescente com câncer e suas principais dificuldades após o início do mesmo, por meio de aplicação de um questionário WHOQOL-bref adaptado às necessidades do presente estudo, pelos pesquisadores, à pacientes em tratamento oncológico em um hospital no estado de São Paulo.

Participaram desse estudo 21 pacientes na faixa etária de 12 a 17 anos, de ambos os sexos e que estavam em tratamento em um instituto de tratamento do câncer de uma cidade no estado de São Paulo. Os critérios de inclusão foram os pacientes em fase de tratamento oncológico nesse instituto em estudo e que pertenciam à faixa etária de adolescentes, que segundo o Estatuto da Criança e do Adolescente (ECA), abrange indivíduos entre 12 e 18 anos, e que estavam abertos para realização do questionário e conversa com os pesquisadores; e os de exclusão foram os pacientes recém diagnosticados com câncer e aqueles em tratamento, mas fora da faixa etária definida.

Os pesquisadores garantiram sigilo absoluto das informações. Nenhum benefício material e financeiro foi oferecido aos participantes e responsáveis legais. Ao que concerne riscos aos participantes, a pesquisa não trouxe nenhum risco notável. Ao que se refere os benefícios, a pesquisa em questão possibilita uma busca por melhorias na promoção à saúde integral, não apenas no âmbito biológico, mas no psicossocial.

Para a análise dos dados obtidos pelos questionários aplicados foram utilizadas ferramentas desenvolvidas a partir do próprio Grupo WHOQOL, realizando uma média dos valores de cada faceta e também um cálculo de cada domínio presente no questionário, através da soma dos valores das facetas de cada domínio dividida pelo número de facetas de determinado domínio.

O Comitê de Ética da Universidade de Mogi das Cruzes aprovou o protocolo do presente estudo com o parecer de número 3.663.086 e com o Certificado de Apresentação de Apreciação Ética de número 22642819.7.0000.5497. Os participantes concordaram em participar e assinaram o Termo de Consentimento Livre e Esclarecido e o Termo de Assentimento que estão em consonância à Resolução 466/12 do Conselho Nacional de Saúde (CNS) e da Norma Operacional no 001 de 2013 do CNS. 


\section{RESULTADOS E DISCUSSÃO}

Em relação aos dados sociodemográficos, a amostra final foi composta por 21 adolescentes em tratamento no Instituto de Tratamento do Câncer Infantil, a faixa etária possuiu variação entre 12 e 17 anos, com média de idade de 14,6 anos, sendo a faixa entre 14 e 17 anos a mais prevalente, com 15 pacientes $(71,4 \%)($ Tabela 1).

Tabela 1 - Características gerais da amostra.

\begin{tabular}{lcc}
\hline Variáveis & Descrição & $\mathbf{n}(\%)$ \\
\hline \multirow{2}{*}{ Sexo } & Feminino & $11(52,3 \%)$ \\
& Masculino & $10(47,6 \%)$ \\
\hline \multirow{2}{*}{ Faixa Etária } & $12-13$ anos & $6(28,6 \%)$ \\
& $14-15$ anos & $9(42,8 \%)$ \\
\multirow{2}{*}{ Estado de Residência } & $16-17$ anos & $6(28,6 \%)$ \\
\hline
\end{tabular}

Fonte: Costa BO, et al., 2021.

Segundo Presti PDF, et al. (2012), a partir dos 10 anos de idade, a incidência do câncer aumenta exponencialmente, sendo que a taxa de diagnóstico é três vezes maior após os 15 anos. No presente estudo, mesmo se tratando de uma única instituição e com uma amostra pequena, na amostra é observado uma prevalência maior de participantes com 15 anos ou mais, representando 11 pacientes $(52,4 \%)$.

Em relação ao gênero, a incidência pode se modificar conforme a idade, sendo que, em crianças e adolescentes ocorre uma predominância masculina (MUTTI CF, et al., 2018). Na amostra, 11 (52,3\%) são do sexo feminino e, $10(47,6 \%)$ do sexo masculino (Tabela 1). Mas por tratar-se de uma única instituição de tratamento de câncer infantil, com uma pequena amostra de pacientes, pode apresentar diferenças com os dados da literatura.

Quanto ao local de residência, 19 (90,4\%) são residentes do estado de São Paulo, enquanto 2 residem em outros estados, sendo eles, Bahia e Minas Gerais (Tabela 1). Porém, pelo estudo ser realizado em uma instituição localização no estado de São Paulo, o número de pacientes procedentes neste pode estar superestimado, não havendo nenhuma relação comprovada do maior risco de desenvolver a doença com o estado de residência.

Segundo o INCA (2021), os cânceres que ocorrem com mais frequência em crianças são diferentes dos observados em adultos, sendo eles: leucemia, tumores cerebrais e da medula espinhal, neuroblastoma, Tumor de Wilms, linfoma, rabdomiossarcoma, retinoblastoma e câncer ósseo. A leucemia corresponde ao câncer mais comum, representando $28 \%$ dos casos, seguido dos tumores cerebrais e da medula espinhal que representam $26 \%$ e do neuroblastoma, com $6 \%$ (ACS, 2019b). A leucemia linfoide aguda foi o câncer mais comum relatado no estudo (19\%) acompanhado do linfoma de Hodgkin (14,28\%), rabdomiossarcoma embrionário (9,52\%) e neuroblastoma (9,52\%) (Figura 1).

Figura 1 - Prevalência dos tipos mais comuns de câncer da amostra $(n=21)$.

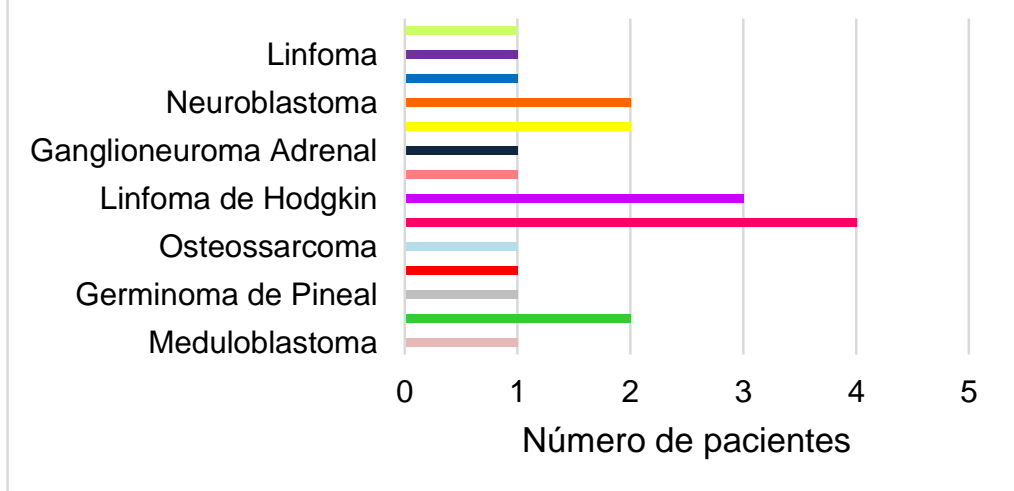

Fonte: Costa BO, et al., 2021. 
Quanto ao tratamento, dos 21 pacientes, $12(57,1 \%)$ realizaram somente quimioterapia, $4(19,1 \%)$ realizaram quimioterapia e radioterapia, 2 (9,5\%) realizaram quimioterapia, radioterapia e cirurgia, $1(4,8 \%)$ realizou quimioterapia e algum procedimento cirúrgico e $2(9,5 \%)$ não haviam realizado nenhum tratamento até a aplicação do questionário (Tabela 2). Um dos principais tratamentos para leucemia linfoide aguda é a quimioterapia e esse dado é confirmado pela correlação entre os dados do tipo de câncer e tratamento, uma vez que todos os pacientes do estudo com o diagnóstico de leucemia linfoide aguda realizaram como tratamento a quimioterapia isolada.

Tabela 2 - Tipo de tratamento dos pacientes

\begin{tabular}{lcc}
\hline Variáveis & Descrição & $\mathbf{n}(\%)$ \\
\hline \multirow{4}{*}{ Tipo de tratamento } & Quimioterapia & $12(57,1 \%)$ \\
& Quimioterapia + radioterapia & $4(19,1 \%)$ \\
& Quimioterapia + cirurgia & $1(4,8 \%)$ \\
Tratamento medicamentoso & Quimioterapia + radioterapia + cirurgia & $2(9,5 \%)$ \\
específico para câncer & Nenhum & $2(9,5 \%)$ \\
& Mercaptopurina & $2(40 \%)$ \\
& Ácido transretinóico (ATRA) & $1(20 \%)$ \\
\hline
\end{tabular}

Fonte: Costa BO, et al., 2021.

O tratamento do câncer não é de domínio total dos oncologistas, ele abrange diversos profissionais da área da saúde (FENGLER AC, et al., 2014). Um dos profissionais presentes nessa equipe multidisciplinar é o farmacêutico que possui papel na seleção de medicamentos, nos esquemas terapêuticos e também para as medidas de suporte para o tratamento, com intuito de minimizar os possíveis efeitos adversos referentes à quimioterapia (LOBATO LC, et al., 2019). Por isso, quando questionados sobre medicamentos em uso, os participantes citaram fármacos que não são utilizados para a doença em propriamente dita, mas para reduzir os efeitos do tratamento oncológico.

Da amostra em estudo, 8 pacientes (38\%) utilizam o Bactrim (Cotrimoxazol), 3 (14,28\%) utilizam o Omeprazol. Enquanto, Gabapentina, Vonau (Ondansetrona), Dexametasona, Dipirona, Carvedilol, Amitrptilina foram citados por 2 (9,5\%) dos pacientes cada e o Montelucaste, Patanol, Baclafen (Baclofeno), Ranitidina, Ácido Fólico, Prednisona, Cálcio, Vitamina D, Lactulona, Risperidona, Valciclovir, Ritalina e Paracetomal foram citados por 1 (4,76\%) paciente cada, apenas.

A sepse representa a principal causa secundária de morte nos pacientes oncológicos. Isso pode ser causado devido à defeitos na quimiotaxia dos neutrófilos, a problemas de fagocitose e à redução da capacidade bactericida. Associa-se a isso a frequente exposição à procedimentos invasivos como sondas, punções, cirurgias e cateteres de longa permanência que aumentam a chance de uma infecção (SILVA MMM, et al., 2022). Nesse sentido, o Cotrimoxazol, que foi o fármaco mais citado pelos participantes da pesquisa, consiste na combinação da trimetoprima com o sulfametazol com diversas indicações terapêuticas como infecções do trato urinário, respiratório, gastrointestinais e para profilaxia para pacientes neutropênicos (HILAL-DANDAN R e BRUNTON LL, 2015).

O tratamento oncológico consiste ainda em um tratamento sistêmico com impacto sobre a divisão das células tumorais, com isso, os efeitos deletérios sobre a divisão das células normais do corpo, como, medula óssea ou trato gastrointestinal, provocam toxicidade que é responsável pelos principais efeitos adversos (CHAVES e GORINI, 2011). Segundo Ramos MB, et al. (2014), náuseas, vômitos e outros sinais de toxicidade gastrointestinais são as principais queixas associadas à quimioterapia sistêmica, o que justifica o uso de medicamentos como o Omeprazol, Ondansetrona e Dexametasona citados pelos participantes do estudo

Em relação ao tratamento medicamentoso específico para o câncer, apenas $5(23,8 \%)$ dos pacientes citaram seu uso, sendo a Mercaptopurina, que é utilizada principalmente no tratamento das Leucemias Linfoides e Mieloides, que corresponde ao tipo de câncer mais comum na amostra, a mais citada (40\%). Depois, o Ácido Transretinóico (ATRA), Irinotecano e Imatinib foi citado por um paciente cada (20\%) (Tabela 2). 
A qualidade de vida é definida como a forma que o indivíduo percebe sua posição na vida, em relação à cultura e sistema de valores, e também, sobre seus objetivos, expectativas, padrões e preocupações (CARVALHO PL, et al, 2013). O adoecimento oncológico possui uma influência negativa principalmente na qualidade de vida do paciente, pois ele passa a viver com uma constante incerteza quanto à evolução da doença e sobrevivência (SILVA APA, et al., 2019).

Alguns parâmetros como atividade física, condições sociais, físicas, psicológicas e sociais, expressam a qualidade de vida de uma pessoa e estes foram avaliados com a utilização do questionário WHOQOL-Bref, com a análise em quatro domínios, que são físicos, psicológico, relações sociais e meio ambiente. E em 26 facetas, sendo 24 divididas entre os domínios e 2 sobre como o indivíduo avalia sua qualidade de vida e sobre quão satisfeito o mesmo está com sua saúde.

Questionados quanto à autoavaliação da qualidade de vida, a maioria, $15(71,4 \%)$ classificaram como "muito boa" ou "boa" enquanto $5(23,8 \%)$ classificaram como "nem ruim, nem boa". Porém, quando questionados o quão satisfeito estavam com a própria saúde, $11(52,3 \%)$ responderam entre "muito satisfeito" ou "satisfeito" enquanto 8 (38\%) responderam "nem satisfeitos, nem insatisfeitos" e $2(9,5 \%)$ responderam "insatisfeitos" resultando em uma regular satisfação com a saúde (Tabela 3). Nesse sentido, pode-se inferir que a maioria dos participantes do estudo avaliam positivamente sua qualidade de vida, mas desta maioria, nem todos estão satisfeitos com sua saúde, o que pode ser atribuído a situação a qual os participantes desta pesquisa estão vivenciando no momento.

Tabela 3 - Escore obtido no domínio de qualidade de vida do WHOQOL-Bref de pacientes em tratamento oncológico $(\mathrm{n}=21)$.

\begin{tabular}{lcccc}
\hline $\begin{array}{c}\text { Item do WHOQOL- } \\
\text { Bref }\end{array}$ & Respostas & $\mathbf{n ~ ( \% )}$ & Média & $\begin{array}{c}\text { Desvio } \\
\text { padrão }\end{array}$ \\
\hline & Muito ruim & 0 & \\
Avaliação da & Ruim & 0 & \\
qualidade de vida (1) & Bom ruim, nem boa & $5(23,8 \%)$ & 4,05 & 0,76 \\
& Muito boa & $9(42,8 \%)$ & & \\
& Não respondeu & $6(28,6 \%)$ & \\
Satisfação com a & Muito insatisfeito & 0 & \\
própria saúde (2) & Insatisfeito & $2(9,5 \%)$ & \\
& Nem satisfeito, nem insatisfeito & $8(38,1 \%)$ & 3,6 & 0,87 \\
& Satisfeito & $8(38,1 \%)$ & & \\
\hline
\end{tabular}

Fonte: Costa BO, et al., 2021.

$\mathrm{Na}$ análise dos resultados dos domínios, foi somado a pontuação de cada domínio em cada faceta e dividido pelo número de facetas, realizando assim a média, conforme proposto. Assim, pelos resultados, classifica-se de 1 até 2,9 uma qualidade de vida que precisa melhorar, de 3 a 3,9 qualidade de vida regular, de 4 a 4,9 qualidade de via boa e 5 uma qualidade de vida muito boa.

A Tabela 4 mostra o número de questionários obtidos em cada classificação, após a realização da média, segundo os quatro domínios do WHOQOL-Bref.

Tabela 4 - Escore obtido nos 4 domínios do WHOQOL-Bref de pacientes em tratamento oncológico ( $\mathrm{n}=21)$.

\begin{tabular}{lcccc}
\hline Classificação/Domínio & Domínio Físico & $\begin{array}{c}\text { Domínio } \\
\text { Psicológico }\end{array}$ & $\begin{array}{c}\text { Relações } \\
\text { Sociais }\end{array}$ & $\begin{array}{c}\text { Meio } \\
\text { Ambiente }\end{array}$ \\
\hline Precisa melhorar & 8 & 5 & 10 & 3 \\
Regular & 7 & 11 & 7 & 9 \\
Boa & 6 & 5 & 4 & 9 \\
Muito Boa & 0 & 0 & 0 & 0 \\
\hline
\end{tabular}

Fonte: Costa BO, et al., 2021. 
Ao serem questionados sobre dor e desconforto; energia e fadiga; sono e repouso; mobilidade; atividades da vida cotidiana; dependência de medicações ou tratamentos; capacidade de trabalho que são critérios englobados no domínio físico, nenhum dos pacientes foram englobados na classificação "muito boa", enquanto $8(38,1 \%)$ "precisam melhorar", 7 (33,3\%) possuem uma qualidade de vida regular e $6(28,6)$ possuem uma "boa" qualidade de vida. Na faceta de avaliação sobre satisfação com o trabalho, por serem adolescentes e a maioria ainda não ter completado a idade mínima para entrada no mercado de trabalho que é de 16 anos, a taxa de respostas em branco foi de 33,3\%, representando a segunda questão com maior número de não respondidos.

$\mathrm{Na}$ avaliação para questões relacionadas com sentimentos positivos e negativos, concentração, pensamentos, memória, autoestima, imagem corporal e aparência, espiritualidade/religião/crenças pessoas, $11(52,4 \%)$ pacientes se enquadram na classificação regular, enquanto $5(23,8 \%)$ estão na classificação boa e os outros $5(23,8 \%)$ precisam melhorar.

Nesse domínio destaca-se a faceta sobre a capacidade de aceitação da aparência física, que de 21 questionários, $15(71,4 \%)$ se aceitam completamente ou possuem muita capacidade de se aceitar enquanto $6(28,3 \%)$ se aceitam médio ou não conseguem se aceitar. Isso porque a adolescência é marcada por alterações físicas por conta da puberdade, uma vez que nessa fase ocorre crescimento de pelos, mudança de voz, desenvolvimento de seios, e quando associado a efeitos colaterais do tratamento quimioterápico como queda de cabelo e o uso de cadeira de rodas e alterações marcantes de peso, as alterações na imagem corporal pode levar ao desenvolvimento de baixa autoestima, visto que nesse período, a aparência física adquire maior destaque do que na infância (SANTOS DBM e OLIVA AD, 2021).

Somado às dificuldades da própria adolescência, o paciente oncológico precisa enfrentar a doença, o tratamento e as preocupações, sentimentos, insatisfação com auto-imagem, isolamento e restrições que podem tornar a sua vida ainda mais difícil. Nesse sentido, ainda dentro do domínio psicológico, a frequência com que os pacientes possuem pensamentos negativos como ansiedade e depressão, merecem destaque, em que $10(95,2 \%)$ dos 21 pacientes já apresentaram tais episódios ou apresentam com frequência, muito frequentemente ou até sempre.

Nas relações sociais e pessoais, apoio social e a vida sexual, 10 (47,6\%) participantes se enquadram na classificação que "precisa melhorar", enquanto 7 (33,4\%) possuem uma qualidade regular e apenas 4 (19\%) podem ser classificados com uma boa qualidade de vida no domínio das relações sociais. $\mathrm{Na}$ análise, destacou-se a faceta sobre a atividade sexual que dentre os 21 questionários, 8 (38\%) não responderam, 9 $(42,8 \%)$ consideraram como "muito ruim" ou "ruim", 1 (4,7\%) como "nem ruim, nem bom" e apenas $3(14,2 \%)$ responderam ser "bom" ou "muito bom". O alto número de questionários em que esse item não foi respondido pode ser explicado pela baixa média de idade dos participantes do estudo em questão. E apesar de que a baixa aceitação da auto-imagem pode levar à vergonha e ao isolamento e assim maior dificuldade nas relações sociais amorosas, pelos dados coletados, não foi possível correlacionar a baixa aceitação da aparência física com a insatisfação com a vida sexual.

No período de tratamento ainda, o adolescente precisa passar por algumas alterações na rotina como períodos longos de internação e isolamento, levando ao afastamento do paciente de vínculos muito importantes como a família, os amigos e a escola. Essa privação de convívio pode gerar sentimentos como tristeza e estresse. (SOUZA RLA, et al., 2021). Por vezes ainda, pode ocorrer um isolamento por parte do próprio paciente devido a drástica mudança de auto-imagem o que causa vergonha e dificuldade de aceitação (FREITAS JAL e OLIVEIRA BLG, 2018). Essa situação pode influir o porquê 17 (80,9\%) dos 21 participantes do estudo foram classificados entre "precisa melhorar" ou "regular" na qualidade das relações sociais.

Questionados sobre segurança, ambiente físico (ruído, poluição, clima, trânsito), recursos financeiros, acesso à informação, oportunidades de recreação/lazer, moradia, serviço de saúde e transporte, que é englobado no domínio do meio ambiente, 9 (42,8\%) pacientes foram classificados como "boa", sendo esse o domínio com maior número de pacientes nessa classificação. Porém, da mesma forma, 9 (42,8\%) pacientes são classificados como regular e 3 (14,4\%) "precisa melhor". Nesse domínio, a faceta que obteve menor 
média foi sobre o quão saudável era o ambiente físico o que é condizente com os dados sociodemográficos dos participantes, já que mais de 90\% residem no estado de São Paulo e destes 57,9\% vivem na cidade de São Paulo, que está entre as 5 cidades mais poluídas do Brasil e é a cidade mais populosa do país.

Quanto à qualidade de vida, verificou-se que o domínio mais comprometido foi o domínio Social, enquanto o mais preservado, é o domínio Meio ambiente. Como foi observado na Tabela 4, a classificação "muito bom" não foi atinigida por nenhum domínio dos 21 questionários obtidos na realização da pesquisa. De forma geral, em relação ao Domínio Físico, Domínio Psicológico e Meio Ambiente classifica-se a qualidade de vida dos pacientes oncológicos entrevistados como "regular", pois obtiveram uma média de 3,25; 3,38 e 3,69 respectivamente. Já em relação ao Domínio Relações Sociais, é visto que a qualidade de vida "precisa melhorar" já que resultou em uma média de 2,84.

Por fim, pela Figura 2, é visto que a maioria, 8 (40\%), dos pacientes oncológicos entrevistados possuem uma qualidade de vida classificada como regular, enquanto $7(31 \%)$ precisam melhorar e apenas $6(29 \%)$ possuem uma qualidade de vida que pode ser classificada como boa. Porém, se for somado a quantidade de pacientes com uma qualidade de vida regular e boa, resultam em $14(69 \%)$ o que é representa o dobro de pacientes de precisam realmente melhorar sua qualidade de vida, resultado esse diferente do esperado em pacientes com uma doença com tratamentos tão agressivos como o câncer, principalmente nessa fase do desenvolvimento, a adolescência.

Figura 2 - Classificação geral da qualidade de vida pelo WHOQOL-Bref dos pacientes oncológicos ( $\mathrm{n}=21)$.

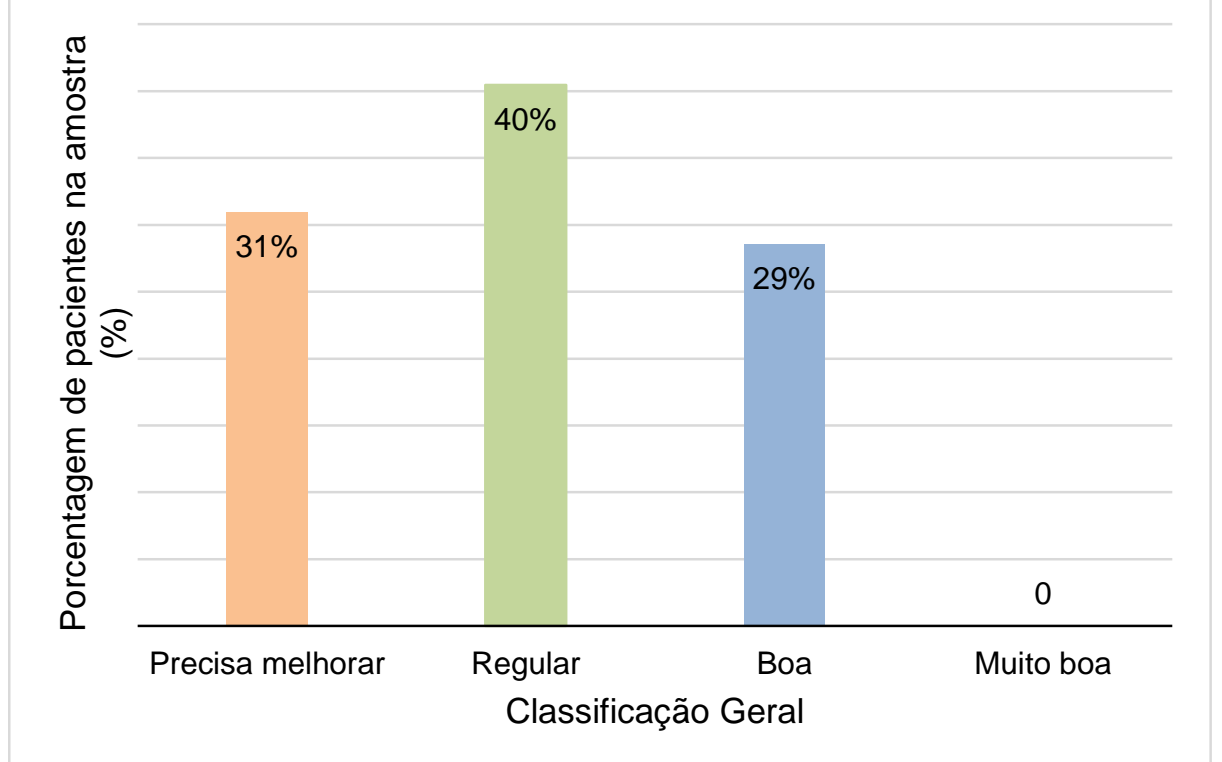

Fonte: Costa BO, et al., 2021.

\section{CONCLUSÃO}

O estudo permitiu perceber que o tratamento quimioterápico excede a sobrevivência dos pacientes, ele visa também a qualidade de vida durante o tratamento, já que ela é uma importante medida de resultados. $O$ conjunto de resultados permitiu visualizar que apesar das dificuldades do diagnóstico e tratamento, de forma geral, o adolescente com câncer não avalia de maneira tão negativa sua qualidade de vida, porém, é mostrado que a ruptura do cotidiano, mudanças de hábitos, imposição de restrição e isolamento, associado à dificuldade de aceitação da própria imagem corporal possuem influência negativa nas relações sociais desses pacientes e consequentemente na qualidade de vida dos mesmos. Com isso, ressalta-se a importância de a equipe multiprofissional trabalhar buscando um tratamento individualizado que engloba além da habilidade técnica e científica, a disponibilidade desses profissionais de escutar, entender e acolher as dificuldades de cada paciente permitindo a criação de um vínculo e uma aproximação com toda equipe o que pode favorecer 0 entendimento, a adesão e o enfrentamento do tratamento. 


\section{REFERÊNCIAS}

1. AMERICAN CANCER SOCIETY (ACS). Risk factors and causes of childhood cancer. 2019a. Disponível em: https://www.cancer.org/cancer/cancer-in-children/risk-factors-and-causes.html. Acessado em: 9 de abril de 2021.

2. AMERICAN CANCER SOCIETY (ACS). Types of Cancer that Develop in Children. 2019b. Disponível em: https://www.cancer.org/cancer/cancer-in-children/types-of-childhood-cancers.html. Acessado em: 9 de abril de 2021.

3. ANJOS C, et al. O câncer infantil no âmbito familiar: Revisão integrativa. Rev. Min. Enferm., 2015; 19(1): $227-233$.

4. CARVALHO PL, et al. Qualidade de vida de mulheres em tratamento de câncer de mama. J. Health Sciences Institute, 2013; 31(2): 187-92.

5. CHAVES PL, GORINI MIPC. Qualidade de vida do paciente com câncer colorretal em quimioterapia ambulatorial. Revista Gaúcha de Enfermagem, 2011; 32(4): 767-73.

6. DUARTE VI, GALVÃO Al. Câncer na adolescência e suas repercussões psicossociais: percepções dos pacientes. Rev. SBPH, 2014; 17(1): 26-48.

7. FARINHAS VG, et al. Impacto Psicológico do Diagnóstico de Câncer na Família: Um Estudo de Caso a Partir da Percepção do Cuidador. Pensando Famílias, 2013; 17(2): 111 - 129.

8. FELICIANO SV, SANTOS MO, POMBO-DE-OLIVEIRA MS. Incidência e Mortalidade por Câncer entre Crianças e Adolescentes: uma Revisão Narrativa. Revista Brasileira de Cancerologia 2018; 64(3): 389-396

9. FENGLER AC, et al. A atuação do farmacêutico no tratamento do paciente oncológico. Universidade Regional do Noroeste do Estado do Rio Grande do Sul, 2014.

10. FREITAS JAL, OLIVEIRA BLG. Aspectos Psicológicos envolvidos na sobrevivência do câncer infantil. Revista Uningá, 2018; 55(2): 1-13.

11. FERENSOVICZ JMR. Vivenciando o câncer na adolescência: uma revisão de literatura. Monografia (Bacharel em Enfermagem) - Centro Universitário Campo Real, Guarapuava -PR, 2020; 36p.

12. HILAL-DANDAN R, RUNTON LL. Manual de Farmacologia e Terapêutica de Goodman \& Gilman. 2ªed. Porto Alegre: AMGH Editora, 2014; 1727-1732p.

13. INSTITUTO NACIONAL DE CÂNCER (INCA). Estimativa/2020: Incidência de câncer no Brasil. 2019. Disponível em: https://www.inca.gov.br/sites/ufu.sti.inca.local/files//media/document//estimativa-2020-incidencia-de-cancer-nobrasil.pdf. Acessado em: 12 de abril de 2021.

14. INSTITUTO NACIONAL DE CÂNCER (INCA). INCA estima quase 600 mil casos novos de câncer para 2016.2015. Disponível em: https://www.inca.gov.br/noticias/inca-estima-quase-600-mil-casos-novos-cancer-para2016\#: :text=O\%20aumento\%20da\%20expectativa\%20de,os\%20brasileiros\%20no\%20pr\%C3\%B3ximo\%20ano. Acessado em: 06 de abril de 2019.

15. INSTITUTO NACIONAL DE CÂNCER (INCA). Tipos de câncer. Câncer Infantojuvenil. 2021. Disponível em: https://www.inca.gov.br/tipos-de-cancer/cancer-infantojuvenil. Acessado em: 09 de abril de 2021.

16. LOBATO LC, et al. Cuidados Farmacêuticos no tratamento oncológico: Uma revisão integrativa da Literatura. Cuidados Farmacêuticos, 2019; 14(1): 31-38.

17. MUTTI CF, et al. Perfil Clínico-epidemiológico de Crianças e Adolescentes com Câncer em um Serviço de Oncologia. Revista Brasileira de Cancerologia, 2018; 64(3): 293-300.

18. PRESTI PDF, et al. Estudo epidemiológico de câncer na adolescência em centro de referência. Revista Paulista de Pediatria, 2012; 30(2): 210-6.

19. RAMOS MB. Manejo dos efeitos adversos dos antineoplásicos em crianças e adolescentes com osteosarcoma: revisão integrativa. Revista enfermagem UFPE, 2014; 8(3): 4150-8.

20. SANTOS DBM, OLIVA AD. Emoções positivas e resiliência na perspectiva de adolescentes com câncer. Revista Brasileira de Terapias Cognitivas, 2021; 17(1): 39-47.

21. SILVA APA, et al. A influência do exercício físico na qualidade de vida de adultos sobreviventes de câncer. Revista Eletrônica Acervo Saúde, 2019; 34: e1501.

22. SILVA MMM, et al. Prevalência e fatores associados à sepse e choque séptico em pacientes oncológicos em terapia intensiva. Revista Brasileira de Enfermagem, 2022; 75(1): e20201338.

23. SOUZA RLA, et al. A hospitalização na percepção de crianças e adolescentes em tratamento oncológico. Revista Gaúcha de Enfermagem, 2021;42: e20200122.

24. VILLA, D. Sentido da vida em adolescentes vivendo com câncer. Trabalho de conclusão de curso. Universidade de Caxias do Sul, Caxias do Sul, 2019; 54p. 